\title{
Zur Akkommodationstätigkeit des Gehörorgans, ein Beitrag zur Physiologie des Ohres.
}

Von Dr. Hugo Weissenberg (Tichau, O.-Schl.), z. Z. Truppenarzt in Striegau (Schl.).

Der Begriff Akkommodation ist uns am besten vertraut aus der Physiologie des Sehorgans, bei dem wir ja hierunter die Finstellung des Auges - durch Muskelzug an der Linse - zum Sehen für die Nähe und Ferne verstehen. Aber auch beim Gehörorgan sprechen wir von Alkkommodation und meinen hiermit seine Anpassung zum Hören von höh ${ }^{\circ}$ ren und tieferen, lauten und leisen Schallschwingungen. Auch hier handelt es sich um Muskelwirkungen, um die des M. tensor tympani und des M. stapedius, von denen man annimmt, daß sio durch Spannung des Trommelfells und der Gehörknöchelkette das Ohr in die jeweilig günstigste Aufnahmefähigkeit bringen; ihre Tätigkeit ist mehr reflektorisch als willkürlich.

Kürzlich machte ich an mir eine Beobachtung, die mir zu beweisen scheint, daß das Gehörorgan doch auch noch eine ganz anders geartete, sehr einfache Fähigkeit der Akkommodation für höhere und tiefere Schall- 
schwingungen besitzt, die, wie mir scheint, bisher der Feststellung entgangen ist; ich habe wenigstens in keinem physiologischen oder otiatrischen Lehrbuche eine dahingehende Ausfïhrung gefunden. Es handelt sich um Folgendes.

Seit Jahren leide ich an einer durch chronischen Mittelohrkatarrh verursach ten Schwerhörigkeit; vor einigen Monaten erfolgte auf dem rechten Ohr eine Besserung der Hörfähigkeit, sodaß ich wieder deutlich das Ticken meiner Taschenuhr hören kann. Im Anfang der Besserung fiel mir nun eine eigenartige Beobachtung auf, für die ich lange Zeit keine Erklärung finden konute: Hielt ich den Kopf nach rechts geneist, dann konnte ich meine Taschenuhr mit dem rechten $\mathrm{Ohr}$ von der Glasdeckelseite hören, aber nicht von der Metalldeckelseite; hielt ich den Kopf nach links geneigt, dann hörte ich sie - mit demselben $\mathrm{Ohr}$ von der Metalldeckelseite, aber nicht von der Glasseite! Endlich gelang es mir, die, wie mir scheint, richtige Erklärung für dieses eigenartige Phänomen zu finden. Das 'licken der Taschenuhr hat, wie ich bald merkte, von der Metallseite aus gehört, eine andere Tonlage als von der Glasseite aus; bei meiner klingt es von der Glasseite aus etwa in der Höhe des eingestrichenen $\bar{b}$, von der Metallseite aus eine Oktave tiefer (b). Wie ich bei Versuchen mit anderen Taschenuhren und bei Normalhörenden sah, zeigen sich hier ähnliche Verhältnisse. Oífenbar geben eben Metall und Glas verschieden hohe Resonanzsch willgungen.

Nun wissen wir ja, daß die Steigbügelplatte von der perilymphatischen Flüssigkeit bedeckt ist; ihr Druck auf die Steigbügelplatte ist natïrlich kei geneigter Kopfhaltung im tiefer stehenden $\mathrm{Ohr}$ größer als im höher stehenden, da dort der Druck durch die Perilymphe und das Gehirngervicht vermehrt ist. Nun erklärt sich also zwauglos meine obige Beobachtung durch die Schlußf,lgerung, daß für die Fortleit.ng von höheren Schallschwingungen der höhere Druck auf die Stapesplatte, für tiefere die geringere Druckbelasturig am geeignetsten ist. Und es bleibt sich dabei gleich, ob man mit der Helmholtzschen Theorie die Gehörknöchelkette als Zuleitungsapparat uder mit der besonders von Zimmermann (Dresden) vertretenen umgekehrt als Dämpfungsvorrichtung ansieht. Diese unterschiedliche Beeinflussung der höheren (kurzwelligen) und tieferen (langwelligen) Schallsch «ingungen läßt, sich vergleichen mit der verschiedentlichen Brechung der Lichtstrahlen, von denen uns ja bekannt ist, daß die kurzwelligen blauen anders gebrschen werden als die langwelligen roten.

Die Sache liegt also so: Bei gerader Kopfhaltung sind beide Ohren eines normal Hörenden gleichmäBig für hohe und tiefe Schallschwingungen akkommodiert, bei Schräghaltung des Kopfes hört das höher stehende die tieferen und das tiefer stehende $\mathrm{Ohr}$ die höheren lautor als beiGradhaltung Von dieserAkkommodation machen wir un bewußtGebrauch beimLauschen, bei dem man ja zumeist uwwillkürlich den Kopf schräg hält. Es ist mir seh wahrscheinlich, daß dem Optimum derWahrnehmbarkeit verschieden hoher Töne ein bestimmter Grad der Schräghaltung des Kopfes entsprirht. Ich habe mir zur Bestimmung der Kupfhaltung eine einfache Meßvorrichtung hergestellt, mit der man sie in Wirkelgraden angeben kann. Die Haltung des Kopfes wird ja kestımmt durch drei Ebenen: die sagittale, frontale und horizontale. Die Sagittalebene kann bei diesem Gegenstand hier außer Betracht bleiben, da ja durch die Drehung des Kopfes der Druck der Perilymphe auf die Steigbügelplatte nicht beeinfluBt wird: sie kann übrigens leicht mit der Magnetnadel bestimmt werden. Die Lageveränderungen der Frontal- und

Horizuntalebenen kann man bestimmen durch ein Lot, dessen Aufhängungspunkt und Ausschlagsskala am Schädel fixiert ist. Die Skala selbst muß, da es sich ja hier um Ausschlage in drei Ébenen handelt, eine Kugelfläche sein, die innen, ähnlich wie beim Globus, in Längen- uad Breitengrade geteilt ist.

Bei dem hier ab gebildeter Modell ist der Kugelradius $9 \mathrm{~cm}$ und dementsprechend die Länge des Lotes etwa $2 \mathrm{~mm}$ kürzer. Die Meß vorrichtung wird in einfacher Weise mit einem Stirnband am Kopfe befestigt. Die Skala wird natürlich nicht wie hier im Bilde mit der Hand festgehalten; hier geschah es nur, weil das Modell aus Gips hergestellt und etwas schwer ist; es soll natürlich aus Pappe oder dünnem, leichtem Metall sein. WelcheArt van Tonquellen außer Taschenuhren sich für diese Untersuchung am besten eignet, bleibt festzustellen.
Stimmgabeln werden wahrscheinlich nicht glut zu benutzen sein; sie haben ja auch sonst, wie auch Prof. Kretschmann in dieser Wochenschrift 1917, S. 66, hervorhebt, für Hörprüfungen nur einen begrenzten Wert. Der Normalhörende kann durch Versuche mit Taschenuhren meine $\mathbf{B e}-$ obachtung nachprüfen; dabei will ich aber hertorheben, daß ich auch
Fig. 2.

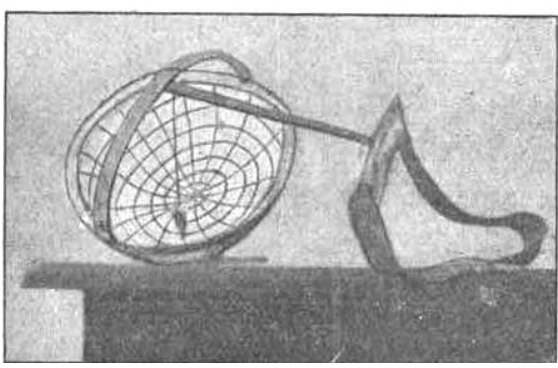
Taschenuhren gefunden hube, die von der $\mathrm{Me}$ tallseite in höherer Tonlage ticken als von der Glasseite und daß der Schwerhörige of tmals bestimm tere Angaben machen karn, weil die Schallstärkedifferenz oft sehr gering ist und für ersteren zu untersicheiden ist zwischen lauter und leiser, was bei der verschiedenen Tonlage nicht immer leicht ist; der Schwerhörige aber hat meistens unr zu un verscheiden zwischen Hören und Nichthören. Bei mir selbst tritt das Phänomen so deutlich in Erscheinung, daß bei Neigung des Kopfes, besonders nach rechts vorn bzw. links hinten, die entsprechende Wahrnehmbarkeit des Uhrtickens von der Metall- bzw. Glasdeckelsęite augenblicklich und unverkennbar wechselt.

Es wird nunmehr bei Hörpriifungen mit der Taschenuhr auf das hier Geschilderte Rücksicht zu nehmen sein; bisher hat nan ja wohl kaum daran gedacht, daß es dabei ein Unterschied sei, ob man die Uhr mit der Metall- oder mit der Glasseite ans. Ohr hält, oder gar, daß die Haltung des Kopfes zu berücksichtigen sei. Vielleicht können durch exakte Bestimmungen der Beziehung von Kopfhaltung zi der Intensität der Wahrnehmbarkeit verschieden hoher Töne diagnostische Schlüsse gezugen werden. Zu berücksichtigen dürfte bei solchen Versuchen sein, da $B$ die Fenestra ovalis nicht in einer der Hauptebenen, sondern in einer Zwischenebene gelegen ist.

Aber auch rein physiologisch betrachtet, scheint mir diese Funktionsteilung beider Ohren, die man. doch bisher für völlig gleichartig ansieht, recht interessant zu sein, besonders auch der Umstand, $\mathrm{daB}$ durch diese antagonistische Wirkung beider Ohren bei Schräghaltung und wechselnder Stellung des Kopfes die dem Hörzentrum im Gehirn zugeführte gesamte Schallintensität duch immer die gleiche bleibt, weil das eine Ohr die höheren bzw. tieferen Töne um so viel stärker hört als das andere schwächer, und umgekehrt.

Nicht fern liegt der Vergleich mit dem stereoskopischen Sehen, bei dem ja das linke Auge auch anders sieht als das rechte, das eine den Gegenstand mehr ron links, das andere mehr von rechts, was aber auch erst bei aufmerksamerem Zusehen zum BewuBtsein kcmmt. Jedenfalls ist wohl das hier beschriebene Phänomen wert, von fachmännischer Seite mit feineren Versuchsapparaten iachgeprüft und bearbeitet $z u$ werden. Man kann es am einfachsten so ausdrücken, daß man sagt, bei schräger Kopfhaltung ist das tiefer stehende $\mathrm{Ohr}$ akkummodiert für höhere, das höher stehende für tiefere Schallschwingungen. 